\title{
New small planetary nebulae discovered in the Galactic center direction
}

\author{
Sławomir K. Górny \\ Copernicus Astronomical Center, Rabiańska 8, 87-100 Toruń,Poland \\ email: skg@ncac.torun.pl
}

\begin{abstract}
During an ongoing search for small planetary nebulae in the southern hemisphere 24 candidates have been found and spectroscopically observed in a region of 45 square degrees.
\end{abstract}

Keywords. planetary nebulae: general, Galaxy: center

\section{Introduction}

We report on an ongoing search for small planetary nebulae $(\mathrm{PNe})$ in the southern hemisphere. It was motivated by the possibility of a statistical bias towards evolved and highly evolved PNe of the known Galactic population after the CD-ROM release of the Edinburgh/AAO/Strasbourg catalogue (Parker et al. 2001/2003). Our program was initially designed to balance it by unveiling the sample of younger, compact and dusty PNe expected to be strong infrared emitters (e.g. Górny et al. 2001) and as an ultimate goal to help investigate different Galactic PNe populations (see e.g. Górny et al. 2004).

To select candidates we use the photometric data and images from the SuperCOSMOS scanned plates of the AAO/UKST H-alpha survey (Parker et al. 2005). The other available broad-band visual images taken at different epochs help to discriminate against variable stars that imitate $\mathrm{H} \alpha$ excess sources. Additional criteria have been developed to select promising PNe candidates from recent infrared and near-infrared surveys (Górny, Omont \& Schultheis, in preparation).

The selected sources are observed with short exposure times using a low resolution spectrograph at the 1.9 meter telescope of the South African Astronomical Observatory.

Table 1. New planetary nebulae candidates.

\begin{tabular}{lcc}
\hline \multicolumn{1}{c}{ PNG } & RA Dec 2000 & obs. date \\
\hline $005.3+02.5$ & $174812.6-225938$ & May 2004 \\
$006.0+05.6^{b}$ & $173825.7-205218$ & Jun 2005 \\
$006.1+02.9$ & $174824.8-221152$ & Apr 2003 \\
$006.1+04.1$ & $174410.6-212921$ & May 2004 \\
$006.2+00.8^{b}$ & $175633.2-231147$ & May 2004 \\
$006.2+06.9^{b}$ & $173413.7-200052$ & Apr 2003 \\
$006.3+01.7^{b}$ & $175328.3-223423$ & May 2004 \\
$006.8+05.0^{c}$ & $174225.9-202638$ & Apr 2003 \\
$007.3+01.7^{b}$ & $175534.7-214238$ & May 2004 \\
$007.3+03.5^{a}$ & $174926.7-205431$ & May 2004 \\
$007.4+01.7^{b}$ & $175542.6-214018$ & May 2004 \\
$007.4+02.5$ & $175242.7-211536$ & May 2004 \\
\hline
\end{tabular}

\begin{tabular}{lcc}
\multicolumn{1}{c}{ PNG } & RA Dec 2000 & obs. date \\
\hline $007.6+02.8$ & $175207.8-205241$ & May 2004 \\
$007.8+04.3^{b}$ & $174715.5-195728$ & Apr 2003 \\
$008.1+01.3^{b}$ & $175840.6-211246$ & May 2004 \\
$008.4+08.8$ & $173206.0-170652$ & Aug 2005 \\
$008.5+02.4$ & $175542.3-202259$ & Aug 2005 \\
$008.6+06.7^{a}$ & $174021.3-180511$ & Aug 2005 \\
$008.8+03.8^{b}$ & $175108.7-192547$ & Apr 2003 \\
$008.8+03.9$ & $175047.3-192104$ & Aug 2005 \\
$008.8+06.2$ & $174232.4-180944$ & Aug 2005 \\
$009.3+02.5$ & $175652.2-193301$ & Aug 2005 \\
$009.4+03.99^{a}$ & $175217.8-185202$ & Aug 2005 \\
$010.0+03.1$ & $175617.3-184236$ & May 2004 \\
\hline & &
\end{tabular}

Comments: a) first published in Boumis et al. (2003, 2006); b) independently discovered by Parker et al. (2006); c) possibly not PN (Parker, private communication). 

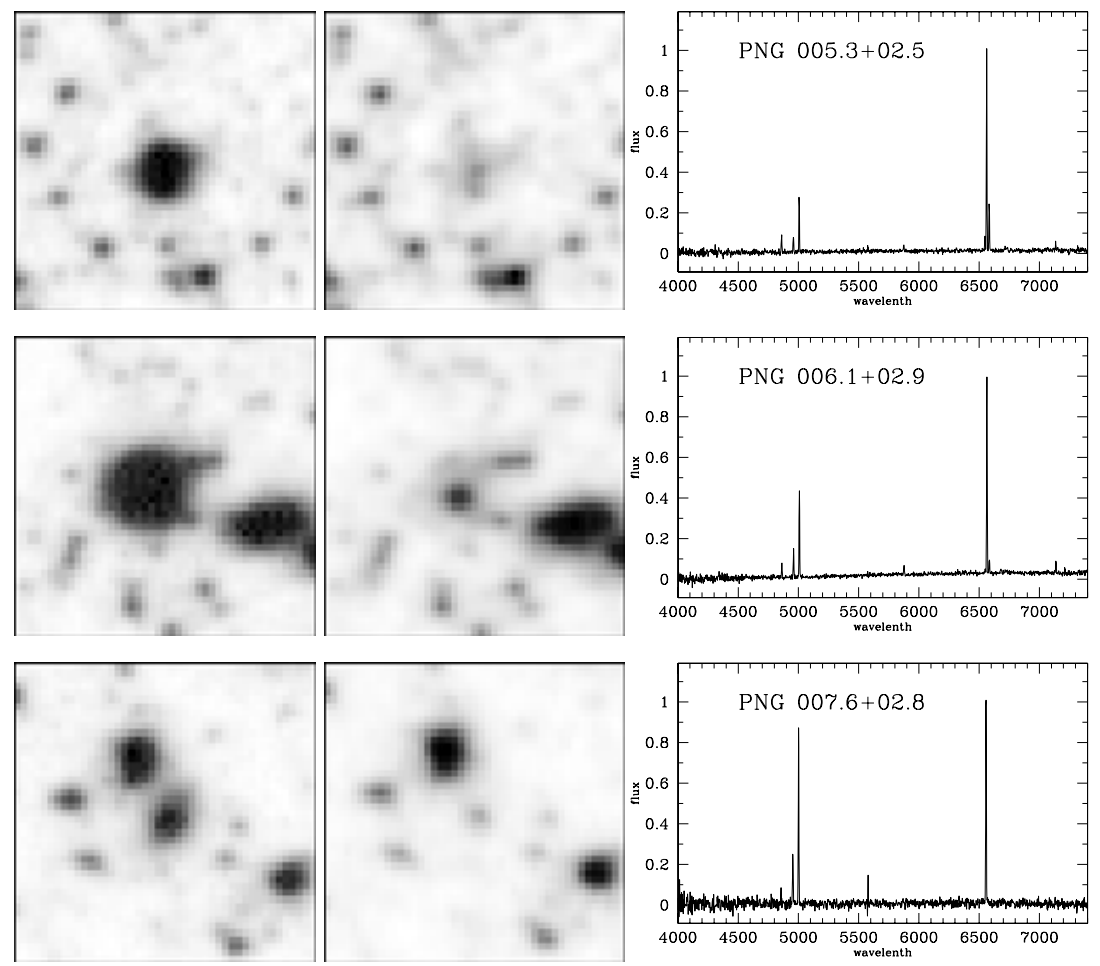

Figure 1. The $\mathrm{H} \alpha$ and $\mathrm{R}$ band images $\left(30^{\prime \prime} \times 30^{\prime \prime}\right)$ and spectra of three small PNe candidates.

\section{Results}

We present here our results from the best observed field near the Galactic center limited by coordinates: $5^{\circ}<l<10^{\circ}$ and $0^{\circ}<b<9^{\circ}$. The $24 \mathrm{PNe}$ candidates found so far in this region are listed in Table 1.

Nine of our $\mathrm{PNe}$ with intermediate diameters have been independently discovered during a recent follow-up program of the AAO/UKST survey (Parker et al. 2006). The remaining objects are all small or star-like in appearance. A few examples are shown in Figure 1 presenting their $\mathrm{H} \alpha$ and $\mathrm{R}$ band images along with the low-resolution spectra.

Our search in this particular Galactic field is already complete down to about 15th magnitude in $\mathrm{H} \alpha$ but we plan to reach weaker sources and should reveal candidates with $\mathrm{E}(\mathrm{B}-\mathrm{V})=4$ located in more dust obscured regions close to the Galactic plane.

\section{Acknowledgements}

This research has partly been supported from a grant No.2.PO3D.002.25 financed by the Polish State Committee for scientific Research and a SALT International Network grant No.76/E-60/SPB/MSN/P-03/DWM 35/2005-2007.

\section{References}

Boumis, P., Paleologou, E.V., Mavromatakis, F., \& Papamastorakis, J. 2003, MNRAS 339, 735

Boumis, P., Akras, S., Xilouris, E.M., et al. 2006, MNRAS 367, 1551

Górny, S.K., Stasińska, G. Escudero, E., \& Costa, R.D.D. 2004, A\& A 427, 231

Górny, S.K., Stasińska, G. Szczerba, R., \& Tylenda, R. 2001, A\&A 377, 1007

Parker, Q.A., Acker, A., Frew, D.J., et al. 2006, MNRAS in press

Parker, Q.A., Hartley, M., Russeil, D., et al. 2003, Proc. IAU Symposium No. 209, p. 41

Parker, Q.A., Phillips, S., Pierce, M.J., et al. 2005, MNRAS 362, 689 